\title{
On the Grundy number of CAMERON gRAPHS
}

\author{
Wing-Kai Hon ${ }^{1}$, Ton Kloks, Fu-Hong Liu ${ }^{1}$, Hsiang-Hsuan Liu ${ }^{1,2}$, and \\ Tao-Ming Wang ${ }^{3}$ \\ 1 National Tsing Hua University, Hsinchu, Taiwan \\ (wkhon, fhliu,hhliu)@cs.nthu.edu.tw \\ 2 University of Liverpool, Liverpool, United Kingdom \\ hhliu@liverpool.ac.uk \\ 3 Tunghai University, Taichung, Taiwan \\ wang@go.thu.edu.tw
}

\begin{abstract}
The Grundy number of a graph is the maximal number of colors attained by a first-fit coloring of the graph. The class of Cameron graphs is the Seidel switching class of cographs. In this paper we show that the Grundy number is computable in polynomial time for Cameron graphs.
\end{abstract}

\section{Introduction}

A proper coloring of a graph is a partition of its vertices into independent sets. We refer to the sets in the partition as color classes, or simply as colors. The chromatic number of a graph $G$, denoted as $\chi(G)$, is the minimal number of colors used in a proper coloring.

Definition 1. Let $\left\{\mathrm{C}_{1}, \ldots, \mathrm{C}_{\mathrm{k}}\right\}$ be the color classes of a proper coloring of $\mathrm{G}$. The coloring is a first-fit coloring if each vertex in color class $C_{j}$ has at least one neighbor in every color class $C_{i}$ with $i<j$.

The maximal number of color classes in a first-fit coloring is called the Grundy number of $G$. We denote the Grundy number as $\Gamma(G)$. Notice that, if $C_{1}, \ldots, C_{k}$ are the color classes of a first-fit coloring, then for each $i, C_{i}$ is a maximal independent set in the subgraph induced by

$$
\bigcup_{j=i}^{k} C_{j} .
$$

This property characterizes first-fit colorings.

A graph is a cograph if it has no induced $\mathrm{P}_{4}$, that is a path with four vertices. Cographs are the graphs that are closed under unions and joins. It is easily seen that in every cograph, every maximal independent set meets every maximal clique. This property characterizes the class of cographs. By means of this characterization, Christen and Selkow prove the following theorem. We give a different proof. 
Theorem 1. When $\mathrm{G}$ is a cograph,

$$
\Gamma(\mathrm{G})=\omega(\mathrm{G})=\chi(\mathrm{G}) .
$$

Proof. Let $\mathrm{G}$ be a cograph. If $\mathrm{G}$ is the union of two smaller cographs, $\mathrm{G}_{1}$ and $\mathrm{G}_{2}$, then

$$
\Gamma(\mathrm{G})=\max \left\{\Gamma\left(\mathrm{G}_{1}\right), \Gamma\left(\mathrm{G}_{2}\right)\right\} .
$$

Assume that $G$ is the join of two smaller cographs, $G_{1}$ and $G_{2}$. Then any independent set has vertices only in one of the two graph $G_{1}$ or $G_{2}$. It follows that

$$
\Gamma(\mathrm{G})=\Gamma\left(\mathrm{G}_{1}\right)+\Gamma\left(\mathrm{G}_{2}\right) .
$$

Notice that the clique number, and also the chromatic number, of G, satisfy recurrences similar to (1) and (2). Since the above exhausts all alternatives, this proves the theorem.

Definition 2. Let $\mathrm{G}$ be a graph and let $\mathrm{S} \subseteq \mathrm{V}(\mathrm{G})$ be a subset of vertices of $\mathrm{G}$. The Seidel switch with respect to $\mathrm{S}$ is the graph obtained from $\mathrm{G}$ by complementing the adjacencies and nonadjacencies of pairs with one element in $\mathrm{S}$ and the other in $\mathrm{V} \backslash \mathrm{S}$.

The interest in the Seidel switch grew out of the observation that the spectrum, that is, the multiset of eigenvalues of the $\{0,-1,1\}$-adjacency matrix remains the same under switching.

Definition 3. A graph is Cameron if it is obtained from a cograph by a Seidel switch.

The Cameron graphs are perfect. They are characterized by a finite set of forbidden induced subgraphs, namely the switching class of $C_{5}$, that is, the $C_{5}$, the bull, gem and co-gem. Another characterization states that a graph is Cameron if switching with respect to the neighborhood of a vertex produces a cograph (with the chosen vertex as an isolated vertex). That follows easily from the fact that the gem and cogem are forbidden, namely, this implies that for each vertex the neighborhood and nonneighborhood induce cographs. The observation above, together with the linear-time recognition of cographs obtained by, eg, Corneil, Perl and Stewart, yields an $\mathrm{O}\left(\mathrm{n}^{2}\right)$ recognition algorithm of Cameron graphs.

Corollary 1. Cameron graphs are recognizable in $\mathrm{O}\left(\mathrm{n}^{2}\right)$ time.

Like cographs, Cameron graphs form a self-complementary class of graphs. Notice that $C_{6}$ and $P_{5}$, but not $P_{6}, C_{5}$ nor any cycle longer than $C_{6}$, are Cameron graphs. The Grundy numbers of $C_{6}, P_{4}$ and $P_{5}$ are 3 so, Theorem 1 is no longer true for Cameron graphs.

Cographs are characterized by the property that every induced subgraph with at least two vertices has a twin, that is, a module with two vertices. Cameron graphs satisfy a similar characterization. Define an anti-twin as a pair of vertices $x$ and $y$ such that every other vertex is adjacent to exactly one of the two. 

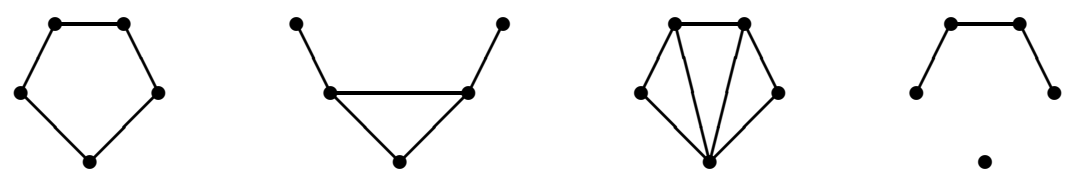

Fig. 1. $\mathrm{A} \mathrm{C}_{5}$, bull, gem and cogem
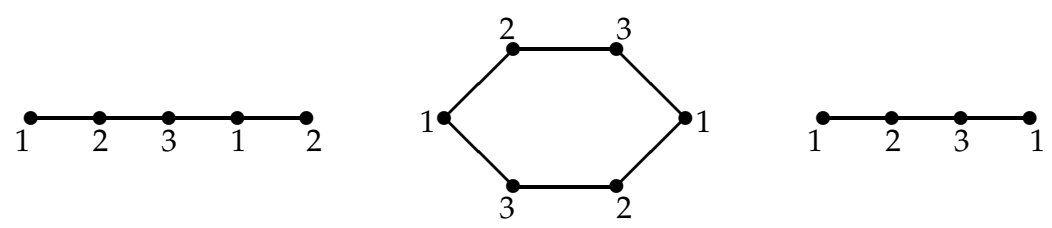

Fig. 2. Grundy coloring of $P_{4}, P_{5}$ and $C_{6}$

Theorem 2. A graph is Cameron if and only if every induced subgraph with at least two vertices has a twin or an anti-twin.

Some remarks on two-graphs $A$ two-graph is a pair $(X, \Delta)$ where $X$ is a set and $\Delta$ is a collection of 3-subsets of $X$ with the property that every 4-subset of $X$ contains an even number of 3 -subsets that are in $\Delta$. For example, when $G$ is a graph with vertex set $X$, then the collection of triples $\Delta$ that have an odd number of edges between them (called the 'odd triples') defines a two-graph $(X, \Delta)$. When two graphs are Seidel switching-equivalent then they yield the same two-graph and, conversely, every two-graph corresponds uniquely to a Seidel switching class of graphs.

Consider the triples in $C_{5}$ with an odd number of edges. Cameron calls this two-graph the pentagon. He characterizes the two-graphs that do not contain the pentagon as an induced substructure as follows. Consider a tree $T$, without vertices of degree two, and let $X$ be the set of leaves of T. Since $T$ is bipartite and connected, it has a unique two-coloring. Call the colors in a two-coloring black and white. Let a set of leaves $\{x, y, z\}$ be a triple of $\Delta$ if the paths connecting the three meet in a black vertex. Then $(X, \Delta)$ is a two-graph and the two-graphs obtained in this manner are exactly the two-graphs without the pentagon as an induced substructure.

In the same papers, Cameron characterizes, and counts, also the two-graphs that don't have a pentagon nor a hexagon as an induced substructure. 


\section{Cameron graphs}

The following characterization is readily checked.

Theorem 3. A graph is Cameron if and only if there exists a coloring of the vertices with colors black and white such that the set of vertices of every nontrivial induced subgraph has a partition into two sets such that all crossing adjacencies are between vertices of the same color or between vertices of opposite colors.

When a Cameron graph is obtained from a cograph via the switching with respect to a set $S$, then coloring all vertices of $S$ white and the remaining vertices black, satisfies the property mentioned in the theorem. For example, let $\mathrm{G}$ is a Cameron graph and let $x$ be any vertex of $G$. Color the vertices of $N(x)$, that is, the neighborhood of $x$, white, and the remaining vertices black. If we switch the graph with respect to the white vertices, we obtain a cograph $H$ in which $x$ is an isolated vertex. Since $\mathrm{H}$ is a cograph it has a binary, rooted decomposition tree, called a cotree. Each leaf of the tree corresponds uniquely to a vertex of the graph. Each internal node (including the root) of this tree is labeled as a join node or a union node. When the node is a join node, each vertex mapped to a leaf in the left subtree is made adjacent to each vertex that is mapped to a leaf in the right subtree. When an internal node of the decomposition tree is labeled as a union node, then no vertex of the left subtree is adjacent to any vertex of the right subtree.

The decomposition tree for the Cameron graph $\mathrm{G}$ is the same rooted binary tree. When an internal node is labeled as a join node for the cograph, then vertices of similar colors in the left and right tree are made adjacent. When an internal node is labeled as a union node, then exactly those pairs of vertices in the left and right subtree that have opposite colors are made adjacent. Henceforth, we refer to the join nodes in the decomposition tree as 'parallel nodes,' and to the union nodes as 'crossing nodes.'

\section{Example}

As an example, we present the following real-world problem. In some faraway country there are $N$ villages, numbered $1, \ldots, N$. In village $i$ there are $b_{i}$ boys and $g_{i}$ girls eligible for marriage. However, albeit a bit archaic, the country's law and culture forbids the marriage of girls and boys that are from the same village. To study population growth, scientists are interested in this question: what is the minimal number of couples that get married, if we don't allow any single boy-and-girl pair from distinct villages.

The Cameron graph $\mathrm{G}$ that represents the problem consists of $\mathrm{N}$ cliques. Clique $i$ consists of $b_{i}$ black vertices and $g_{i}$ white vertices. Between any two cliques $i$ and $j$ we have a parallel connection, that is, all black vertices of clique $i$ are adjacent to all black vertices of clique $j$ and all white vertices of clique $i$ 
are adjacent to all white vertices of clique $j$. (Only heterosexual marriages are allowed. Thus, the Cameron graph is cobipartite.)

We are interested in the Grundy number of this Cameron graph. Notice that each independent set consists either of a single vertex or, of a black and white vertex from different cliques. The minimal number of pairs that get married is therefore,

$$
|\mathrm{V}(\mathrm{G})|-\Gamma(\mathrm{G}) .
$$

It remains to show that we can compute $\Gamma(\mathrm{G})$ in polynomial time.

Construct the decomposition tree for G; this is a rooted binary tree with the black and white vertices in the leaves. Each internal vertex is labeled as a parallel node or a crossing node. In this example, we can have a decomposition tree with one crossing node for each village, that node connects all the boys and girls from that same village. We may assume that all the other internal nodes are parallel nodes.

Our method to solve this problem is a dynamic programming on this decomposition tree. For an internal node $t$ denote the set of vertices mapped to the leaves in the subtree by $V_{t}$. The algorithm computes a boolean function

$$
\tau\left(b_{t}, g_{t}, b_{t}^{\prime}, g_{t}^{\prime}\right)
$$

which is true if

(i) exactly $b_{t}$ single boys and $g_{t}$ single girls from $V_{t}$ will, presumably, get married with boys and girls from $V(G) \backslash V_{t}$ (they marry in the future), and

(ii) $b_{t}^{\prime}$ boys and $g_{t}^{\prime}$ girls from $V_{t}$ stay single altogether.

The computation of the function $\tau$ for each village is easy; in the remainder we consider internal nodes of the decomposition tree such that each village is either fully contained in the leaves of the left subtree, or it is fully contained in the leaves of the right subtree, or it has an empty intersection with the leaves in the subtree 4

We may assume the following principle of optimality; for any node $t$ in the decomposition tree, either $b_{t}^{\prime}=0$ or $g_{t}^{\prime}=0$ or all these boys and girls are from a single village. For parameters such that this condition cannot be fulfilled, we let

$$
\tau=\text { false. }
$$

\footnotetext{
${ }^{4}$ That is, the decomposition tree represents a laminar family of subsets with the villages as atoms.
} 
Consider an internal node $t$, and consider a parameter set $\left\{b_{\ell}, g_{\ell}, b_{\ell}^{\prime}, g_{\ell}^{\prime}\right\}$ for the left subtree and a parameter set $\left\{b_{r}, g_{r}, b_{r}^{\prime}, g_{r}^{\prime}\right\}$ for the right subtree. We assume that the function $\tau$ evaluates as true for the parameters in the left subtree and for the parameters in the right subtree. The resulting set of parameters

$$
\left\{b, g, b^{\prime}, g^{\prime}\right\}
$$

for the node $t$ is then valid if there exist numbers $\alpha$ and $\beta$ such that $\alpha$ boys on the left get married to $\alpha$ girls on the right and $\beta$ girls on the left get married to $\beta$ boys on the right. Thus, these numbers must satisfy

$$
0 \leqslant \alpha \leqslant \min \left\{b_{\ell}, g_{r}\right\} \text { and } 0 \leqslant \beta \leqslant \min \left\{g_{\ell}, b_{r}\right\} \text {, }
$$

and the resulting set of parameters then equals

1. $b=b_{r}+b_{\ell}-\alpha-\beta$,

2. $g=g_{r}+g_{\ell}-\alpha-\beta$,

3. $\mathrm{b}^{\prime}=\mathrm{b}_{\ell}^{\prime}+\mathrm{b}_{\mathrm{r}}^{\prime}$ and

4. $g^{\prime}=g_{\ell}^{\prime}+g_{r}^{\prime}$.

The optimality condition requires that $b^{\prime}=0$ or $g^{\prime}=0$ or that these singles are all from the same village. By the assumption that each village is either fully contained in a subtree, or disjoint from that subtree, this implies that we must have

(a) $\mathrm{b}^{\prime}=\mathrm{b}_{\ell}^{\prime}$ and $\mathrm{g}^{\prime}=\mathrm{g}_{\ell}^{\prime}$ and $\mathrm{b}_{\mathrm{r}}^{\prime}=\mathrm{g}_{\mathrm{r}}^{\prime}=0$, or

(b) $\mathrm{b}^{\prime}=\mathrm{b}_{\mathrm{r}}^{\prime}$ and $\mathrm{g}^{\prime}=\mathrm{g}_{\mathrm{r}}^{\prime}$ and $\mathrm{g}_{\ell}^{\prime}=\mathrm{b}_{\ell}^{\prime}=0$, or

(c) $b^{\prime}=b_{\ell}^{\prime}+b_{r}^{\prime}$ and $g^{\prime}=g_{\ell}^{\prime}=g_{r}^{\prime}=0$, or

(d) $\mathrm{g}^{\prime}=\mathrm{g}_{\ell}^{\prime}+\mathrm{g}_{\mathrm{r}}^{\prime}$ and $\mathrm{b}^{\prime}=\mathrm{b}_{\ell}^{\prime}=\mathrm{b}_{\mathrm{r}}^{\prime}=0$.

At the root of the decomposition tree we require that

$$
\mathrm{b}=\mathrm{g}=0,
$$

because these singles won't have any opportunity to marry in the future, that is, with singles outside $V(G)$. The answer to the problem, that is, the minimal number of married couples is therefore

$$
\min \left\{\frac{|\mathrm{V}(\mathrm{G})|-\mathrm{b}^{\prime}-\mathrm{g}^{\prime}}{2} \mid \tau\left(0,0, \mathrm{~b}^{\prime}, \mathrm{g}^{\prime}\right)=\text { true }\right\} .
$$

This shows that the Grundy number for Cameron graphs in this example is computable in polynomial time. In the following section we discuss the general case. 


\section{The Grundy number of Cameron graphs}

Let $\mathrm{G}$ be a Cameron graph with a black-and-white coloring and let $\mathrm{H}$ be the cograph that results from the Seidel switch of $G$ with respect to the set of white vertices. Notice that we may assume that $\mathrm{G}$ is connected and that $\overline{\mathrm{G}}$ is connected, since otherwise $\mathrm{G}$ is a cographs and we are done, by Theorem 1 on page 2

Consider an internal node $t$ of the decomposition tree. We refer to $V_{t}$ as the vertices that are mapped to leaves in the subtree rooted at $t$. The set of black and white vertices of $V_{t}$ are denoted as $B_{t}$ and $W_{t}$. similar as in the example, we store information of partial colorings of an internal node $t$ in a boolean function

$$
\tau\left(m_{t}, b_{t}, w_{t}, b_{t}^{\prime}, w_{t}^{\prime}\right)
$$

Here

1. $m_{t}$ is the number of mixed color classes, that is, color classes of $G\left[V_{t}\right]$ that contain at least one black and one white vertex;

2. $b_{t}$ is the number of color classes that consist of only black vertices, and that will, presumably, 'marry' (unite with white color classes) in the future;

3. $w_{\mathrm{t}}$ is the number of color classes that consist of only white vertices and that will, presumably, marry in the future;

4. $b_{t}^{\prime}$ is the number of black color classes that will stay forever single, and

5. $w_{\mathrm{t}}^{\prime}$ is the number of white color classes that will stay forever single.

We assume that there is an ordering of these color classes such that the subsets of the colors on $G\left[B_{t}\right]$ and $G\left[W_{t}\right]$ form a first-fit coloring. Consider the case where the first color class $C$ is of mixed type. Then $C \cap B_{t}$ and $C \cap W_{t}$ are maximal independent sets in $G\left[B_{t}\right]$ and $G\left[W_{t}\right]$. Notice that this implies that $C$ is a maximal independent set in $\mathrm{G}$ (because every internal node of the decomposition tree is a parallel node or a crossing node).

We discuss the updating procedures. Consider a crossing node t. Let

$$
\left\{m_{\ell}, b_{\ell}, w_{\ell}, b_{\ell}^{\prime}, w_{\ell}^{\prime}\right\} \text { and }\left\{m_{r}, b_{r}, w_{r}, b_{r}^{\prime}, w_{r}^{\prime}\right\}
$$

be sets of parameters for the left and right child for which $\tau$ evaluates as true. Then, if we denote the set parameters of $t$ by $m, b, w, b^{\prime}$ and $w^{\prime}$ we have

1. $m=m_{\ell}+m_{r}$,

2. $b=\max \left\{b_{\ell}, b_{r}\right\}$,

3. $w=\max \left\{w_{\ell}, w_{\mathrm{r}}\right\}$,

4. $b^{\prime}=\max \left\{b_{\ell}^{\prime}, b_{r}^{\prime}\right\}$,

5. $w^{\prime}=\max \left\{w_{\ell}^{\prime}, w_{\mathrm{r}}^{\prime}\right\}$. 
For the parallel nodes, the updates are similar as in the example of Section 3 . Assume that $\alpha$ black color classes on the left marry to $\alpha$ white color classes on the right and that $\beta$ white color classes on the left marry with $\beta$ black color classes on the right. Then a necessary condition for the numbers $\alpha$ and $\beta$ is that

$$
\alpha \leqslant \min \left\{b_{\ell}, w_{r}\right\} \text { and } \beta \leqslant \min \left\{w_{\ell}, b_{r}\right\} .
$$

The new parameters become

1. $m=m_{\ell}+m_{r}+\alpha+\beta$,

2. $b=b_{\ell}+b_{r}-\alpha-\beta$,

3. $w=w_{\ell}+w_{\mathrm{r}}-\alpha-\beta$,

4. $\mathrm{b}^{\prime}=\mathrm{b}_{\ell}^{\prime}+\mathrm{b}_{\mathrm{r}}^{\prime}$, and

5. $w^{\prime}=w_{\ell}^{\prime}+w_{r}^{\prime}$.

The optimality condition requires that $b^{\prime}=0$ or that $w^{\prime}=0$ or that all these monochromatic color classes are from the same parallel component. That is, we must have (similar as in the example):

(a) $\mathrm{b}^{\prime}=\mathrm{b}_{\ell}^{\prime}$ and $\mathrm{g}^{\prime}=\mathrm{g}_{\ell}^{\prime}$ and $\mathrm{b}_{\mathrm{r}}^{\prime}=\mathrm{g}_{\mathrm{r}}^{\prime}=0$, or

(b) $\mathrm{b}^{\prime}=\mathrm{b}_{\mathrm{r}}^{\prime}$ and $\mathrm{g}^{\prime}=\mathrm{g}_{\mathrm{r}}^{\prime}$ and $\mathrm{g}_{\ell}^{\prime}=\mathrm{b}_{\ell}^{\prime}=0$, or

(c) $\mathrm{b}^{\prime}=\mathrm{b}_{\ell}^{\prime}+\mathrm{b}_{\mathrm{r}}^{\prime}$ and $\mathrm{g}^{\prime}=\mathrm{g}_{\ell}^{\prime}=\mathrm{g}_{\mathrm{r}}^{\prime}=0$, or

(d) $g^{\prime}=g_{\ell}^{\prime}+g_{r}^{\prime}$ and $b^{\prime}=b_{\ell}^{\prime}=b_{r}^{\prime}=0$.

At the root, we are only interested in the sets of parameters with

$$
\mathrm{b}=w=0,
$$

since there is no opportunity for these color classes to marry in the future, and so, the Grundy number is,

$$
\Gamma(\mathrm{G})=\max \left\{\mathrm{m}+\mathrm{b}^{\prime}+w^{\prime} \mid \tau\left(\mathrm{m}, 0,0, \mathrm{~b}^{\prime}, w^{\prime}\right)=\text { true }\right\} .
$$

Theorem 4. There exists a polynomial-time algorithm that computes the Grundy number of Cameron graphs.

Proof. We prove first that there is a first-fit coloring with the computed set of parameters.

Consider a crossing node $t$. Let parameters for the left and right subtree be

$$
\left\{m_{\ell}, b_{\ell}, w_{\ell}, b_{\ell}^{\prime}, w_{\ell}^{\prime}\right\} \text { and }\left\{m_{r}, b_{r}, w_{r}, b_{r}^{\prime}, w_{r}^{\prime}\right\} .
$$

We may assume that there are partial first-fit colorings for the graphs in the left and right subtree. The parameter setting for the node $t$ is

(1) $m=m_{\ell}+m_{r}$; 
(2) $b=\max \left\{b_{\ell}, b_{r}\right\}$;

(3) $w=\max \left\{w_{\ell}, w_{\mathrm{r}}\right\}$;

(4) $\mathrm{b}^{\prime}=\max \left\{\mathrm{b}_{\ell}^{\prime}, \mathrm{b}_{\mathrm{r}}^{\prime}\right\}$;

(5) $w^{\prime}=\max \left\{w_{\ell}^{\prime}, w_{r}^{\prime}\right\}$.

We claim that that there are colorings for the subgraphs on the left and right that start with the mixed color classes. We prove that below. Then the claim follows easily; any mixed color class that starts a coloring on the left or right is a maximal independent set in $G\left[V_{t}\right]$. This proves that there exists a coloring for $G\left[V_{t}\right]$ that starts with $\mathrm{m}$ mixed color classes.

Consider removing all mixed color classes. The remaining graphs on the left and right, induced by the black and white vertices are cographs. By Theorem 1 , the number of monochromatic color classes is equal to the chromatic number of the respective cographs. For the node $t$, after removal of the mixed color classes, the formulas are the formulas that compute the chromatic numbers of the black and white cographs (which are united, since $t$ is a crossing node; so the formulas are given by (1) on Page 2).

Consider a parallel node t. Consider colorings for the left and right subgraph as in Equation (4). By induction, there exist colorings for the left and right subgraph that start with the mixed color classes (if any). Consider removing the mixed color classes and let $V_{t}^{\prime}$ be the remaining set of vertices of $V_{t}$. Consider numbers $\alpha$ and $\beta$ with

$$
\alpha \leqslant \min \left\{b_{\ell}, w_{r}\right\} \text { and } \beta \leqslant \min \left\{w_{\ell}, b_{r}\right\} .
$$

Take the first $\alpha$ black color classes of the graph on the left and unite them with the first $\alpha$ white color classes on the right. This produces $\alpha$ new, mixed color classes. Notice that these can start a first-fit coloring in graph induced by $V_{t}^{\prime}$.

It is readily checked that we may assume the optimality condition. There can be no unmarried black and white pair, which are in different parallel components.

Consider a first-fit coloring of G. Consider a node $t$; by induction we may assume that the coloring induces partial colorings for the left and right subgraph. That is, the mixed color classes start the coloring on the left and right, and the monochromatic color classes form a coloring of the cographs, induced by the black and white vertices, with $\chi$ colors. If the node $t$ is crossing, the monochromatic color classes need to unite, since otherwise they are not maximal. In case $t$ is a parallel node, notice that the monochromatic color classes on the left and right need to be maximal, otherwise they do not form proper first-fit color classes.

This proves the theorem. 


\section{Supplementary information on Grundy colorings}

A complete coloring of a graph is a coloring such that for every pair of colors, there exists an edge whose endpoints' colors match the colors of the pair. In other words, the union of no two color classes is an independent set. The maximal number of colors in a complete coloring is called the achromatic number and it is usually denoted as $\Psi(\mathrm{G})$. This coloring owes its name, 'complete' coloring, to the homomorphism $G \rightarrow K_{k}$, where $k=\Psi(G)$ is the maximal $k$ for which such a homomorphism exists. Computing the achromatic number is NPcomplete, even for trees and for trivially perfect graphs (which are the graphs without induced $\mathrm{P}_{4}$ and $\mathrm{C}_{4}$; so they include the cographs). Obviously, we have

$$
\chi(G) \leqslant \Gamma(G) \leqslant \Psi(G) .
$$

Interestingly, the achromatic number is fixed-parameter tractable, that is, there exists a constant $c$ and a function $f: \mathbb{N} \rightarrow \mathbb{N}$ such that for each $k \in \mathbb{N}$, the question whether $\Psi(G) \geqslant k$ can be decided in $O\left(f(k) \cdot n^{c}\right)$ time. As far as we know, the question whether the Grundy number is fixed-parameter tractable is open.

Zaker showed that any graph with $\Gamma(\mathrm{G}) \geqslant k$ has an induced subgraph with at most $2^{k-1}$ vertices and with Grundy number at least $k$. This is called a $k$ witness. The existence of a $k$-witness implies that there is an algorithm that runs in $\mathrm{O}\left(\mathrm{n}^{2^{k-1}}\right)$ time to decide if $\Gamma(\mathrm{G}) \geqslant k$.

Zaker shows that computing $\Gamma(\mathrm{G})$ is NP-complete for co-bipartite graphs. Obviously, $\Gamma(\mathrm{G}) \leqslant \Delta(\mathrm{G})+1$. According to Havet and Sampaio, deciding whether $\Gamma(\mathrm{G}) \leqslant \Delta(\mathrm{G})$ is NP-complete for bipartite graphs. Deciding if $\Gamma(\mathrm{G}) \geqslant \Delta(\mathrm{G})-\mathrm{k}$ is fixed-parameter tractable with respect to the parameter $\mathrm{k}$. Bonnet et al. show that the Grundy number is fixed-parameter tractable for chordal graphs, clawfree graphs and graphs excluding a fixed minor.

For cographs, $\Gamma(\mathrm{G})=\chi(\mathrm{G})$. Zaker showed that deciding whether $\Gamma(\mathrm{G})=$ $\chi(\mathrm{G})$ is co-NP-complete. Tang et al. mention the following conjecture by Zaker, which we repeat here because we think it is interesting (apparently, Zaker did not publish this conjecture).

Conjecture 1. If $\mathrm{G}$ is $\mathrm{C}_{4}$-free then $\Gamma(\mathrm{G}) \geqslant \delta(\mathrm{G})+1$, where $\delta(\mathrm{G})$ is the minimal degree of $\mathrm{G}$.

\section{References}

1. Bellman, R., Dynamic programming, Dover, 2003.

2. Bodlaender, H., Achromatic number is NP-complete for cographs and interval graphs, Information Processing Letters 31 (1989), pp. 135-138.

3. Bonnet, E., F. Foucaud, E. Kim and F. Sikora, Complexity of Grundy coloring and its variants, Proceedings COCOON'15, Springer-Verlag, LNCS 9198 (2015), pp. 109-120. 
4. Cameron, P., Two-graphs and trees, Discrete Mathematics 127 (1994), pp. 63-74.

5. Cameron, P., Counting two-graphs related to trees, The Electronic Journal of Combinatorics 2 (1995), \#R4.

6. Clarke, N., S. Finbow, S. Fitzpatrick, M. Messinger, R. Milley and R. Nowakowski, A note on the Grundy number and graph products, Discrete Applied Mathematics 202 (2016), pp. 1-7.

7. Cohen-Addad, V., M. Habib, F. de Montgolfier, Algorithmic aspects of switch cographs, Discrete Applied Mathematics 200 (2016), pp. 23-42.

8. Christen, C. and S. Selkow, Some perfect coloring properties of graphs, Journal of Combinatorial Theory, Series B 27 (1979), pp. 49-59

9. Erdős, P., W. Hare, S. Hedetniemi and R. Laskar, On the equality of the Grundy and ochromatic number of a graph, Journal of Graph Theory 11 (1987), pp. 157-159.

10. Gyárfás, A. and J. Lehel, Effective on-line coloring of $\mathrm{P}_{5}$-free graphs, Combinatorica 11 (1991), pp. 181-184.

11. Havet, F. and L. Sampaio, On the Grundy and b-chromatic numbers of a graph, $\mathrm{Al}$ gorithmica 65 (2013), pp. 885-899.

12. Hung L., T. Kloks and F. Villaamil, Black-and-white threshold graphs. Manuscript on arXiv:1104.3917, 2011.

13. Kierstead, H., D. Smith and W. Trotter, First-fit coloring on interval graphs has performance ratio at least 5. Manuscript on arXiv:1506.00192. 2015.

14. Kloks, T. and Y. Wang, Advances in Graph Algorithms. Manuscript on ViXra:1409.0165, 2014.

15. Mallows, C. and N. Sloane, Two-graphs, switching classes and Euler graphs are equal in number, SIAM Journal on Applied Mathematics 28 (1975), pp. 876-880.

16. Manlove, D. and C. McDiarmid, The complexity of harmonious coloring of trees, Discrete Applied Mathmatics 57 (1995), pp. 133-144.

17. Máté, A., A lower estimate for the achromatic number of irreducible graphs, Discrete Mathematics 33 (1981), pp. 171-183.

18. Montiel, C. R., A new characterization of trivially perfect graphs, Electronic Journal of Graph Theory and Applications 3 (2015), pp. 22-26.

19. Seidel, J., A survey of two-graphs, Colloquio Internazionale sulle Teorie Combinatorie, Rome, 1973, Vol. I, Atti dei Convegni Lincei, No.17 Accademia Nazionale dei Lincei, Rome (1976), pp. 481-511.

20. Seidel, J., Geometry and combinatorics. In (Corneil, Mathon eds.) Selected works of J. J. Seidel, Academic Press, London, 1991.

21. Smorodinsky, S., A note on the online first-fit algorithm for coloring k-inductive graphs, Information Processing letters 109 (2008), pp. 44-45.

22. Tang, Z., B. Wu, L. Hu and M. Zaker, More bounds for the Grundy number of graphs. Manuscript on arXiv:1507.01080, 2015.

23. Telle, J. and A. Proskurowski, Algorithms for vertex partitioning problems on partial k-trees, SIAM Journal on Discrete Mathematics 10 (1997), pp. 529-550.

24. Zaker, M., Results on the Grundy chromatic number of graphs, Discrete Mathematics 306 (2006), pp. 3166-3173.

25. Zaker, M., Inequalities for the Grundy chromatic number of graphs, Discrete Applied Mathematics 155 (2007), pp. 2567-2572. 